\title{
RESEARCH
}

Open Access

\section{Apical root resorption during orthodontic treatment with aligners? A retrospective radiometric study}

Elena Krieger ${ }^{1 *}$, Thomas Drechsler ${ }^{2}$, Irene Schmidtmann ${ }^{3}$, Collin Jacobs ${ }^{1}$, Simeon Haag ${ }^{1}$ and Heinrich Wehrbein ${ }^{1}$

\begin{abstract}
Introduction: Objective of this study was to investigate the incidence and severity of apical root resorptions (ARR) during orthodontic treatment with aligners.

Materials and methods: The sample comprised 100 patients (17-75 years of age) with a class I occlusion and anterior crowding before treatment, treated exclusively with aligners (Invisalign ${ }^{\oplus}$, Align Technologies, Santa Clara, (A, USA). The following teeth were assessed: upper and lower anterior teeth and first molars. Root and crown lengths of a total of 1600 teeth were measured twice in pre- and post-treatment panoramic radiographs. Afterwards, relative changes of the root length during treatment were calculated by a root-crown-ratio taking preand post-treatment root and crown lengths into consideration. A reduction of this ratio was considered as a shortening of the initial root length. Additionally, tooth movements of the front teeth were assessed by lateral cephalograms and the 3-dimensonal set up of each patient.
\end{abstract}

Results: All patients had a reduction of the pre-treatment root length with a minimum of two teeth. On average 7.36 teeth per patient were affected. 54\% of 1600 measured teeth showed no measurable root reduction. A reduction of $>0 \%-10 \%$ of the pre-treatment root length was found in $27.75 \%$, a distinct reduction of $>10 \%-20 \%$ in $11.94 \%$. $6.31 \%$ of all teeth were affected with a considerable reduction of $>20 \%$. We found no statistically significant correlation between relative root length changes and the individual tooth, gender, age or sagittal and vertical orthodontic tooth movement; except for extrusion of upper front teeth, which was considered as not clinical relevant due to the small amount of mean $4 \%$ ARR.

Conclusions: The present study is the first analyzing ARR in patients with a fully implemented orthodontic treatment with aligners (i.e. resolving anterior crowding). The variety was high and no clinical relevant influence factor could be detected. A minimum of two teeth with a root length reduction was found in every patient. On average, 7.36 teeth per patient were affected.

Keywords: Apical root resorption, Thermoplastic appliances, Aligners, Orthodontic, Tooth movement, Anterior crowding

\footnotetext{
* Correspondence: elena.krieger@unimedizin-mainz.de

'Department of Orthodontics, Medical Centre of the Johannes-Gutenberg-University

Mainz, Augustusplatz 2, 55131 Mainz, Germany

Full list of author information is available at the end of the article
} 


\section{Introduction}

Root resorptions (RR) appear to be multifactorial and might be a combination of mechanical effects, a genetic disposition and an individual biological variability [1]. They are described as a permanent loss of tooth structure from the root apex and the clinical manifestation among orthodontic patients is highly variable [2]. Former publications referred that orthodontically treated patients were more likely to gain severe apical root resorption (ARR) [3-5].

Regarding influence factors, Weltman et al. [1] reported in their systematic review comprehensive orthodontic treatment could cause increased incidence and severity of $R R$, and heavy forces might be particularly harmful. Therefore, they recommended the use of light forces especially for intrusion of anterior teeth [1]. The incidence of ARR in incisors seems to be higher than in other teeth and more severely [6-10]. Recent studies investigated the incidence and severity of ARR in patients treated with multibracket appliances, assessing the ARR in bone beam computed tomography (CBCT) [11,12]. They reported about most significant ARR and highest frequencies in incisors and first molars [11] or a significant association between the upper dentition and anterior teeth with the degree of root shortening [12]. Factors like archwire sequencing, bracket prescription, and self-ligation seemed not affecting ARR [1]. Also seemed previous trauma or tooth morphology unlikely being a causative factor [1].

Considering genetic factors influencing the incidence of ARR, Sehr et al. [13] reported that local rather than systemic or genetic factors seemed to have predisposed the subjects of their sample to ARR. Other publications of animal experimental or human studies found various candidate genes potentially directly linked to the development of severe root resorption $[2,8,14]$. Harris et al. [15] reported about a proportion of the hereditary component of $60-80 \%$ for ARR in patients undergoing orthodontic treatment.

Regarding the incidence of ARR and orthodontic treatment with removable thermoplastic appliances (aligners) there are only three publications available [16-18]. At first, a relation between aligner-therapy and RR was described in the case report of Brezniak and Wasserstein [16]. A 25-year-old patient sustained apical root resorption (ARR) of the upper incisors during treatment with aligners. Also 2008, Barbagallo et al. [17] compared in a prospective study the effects of removable thermoplastic appliances with light and heavy orthodontic forces on premolar cementum with a treatment period of eight weeks. They reported that the aligner group had similar but slightly greater RR than the light-force group or approximately six times greater than the untreated control group [17]. Then, Sombuntham et al. examined 2009 aligners and RR in an animal experimental study [18]. Clear plastic appliances were inserted and bonded to the residual dentition in rats for a maximum of seven days [18]. They found histological changes of the periodontal ligament and initial superficial RR [18].

Therefore, this is the first study examining the incidence and severity of ARR in patients, who underwent a fully implemented orthodontic treatment with aligners. A further aim of the present study was to evaluate, if any external factor like age or gender could be found that had an influence on ARR.

\section{Material and methods \\ Study design and subjects}

The sample comprised 100 healthy patients, who were treated exclusively with removable thermoplastic appliances, i.e. aligners (Invisalign ${ }^{\circ}$, Align Technologies, Santa Clara, CA, USA). The treatment was performed by one specialist for orthodontics in one private practice. The sample was selected retrospectively from a larger pool of patients by using patient documentation and based on the following inclusion criteria: class I occlusion with an anterior crowding (arch length discrepancy $<8 \mathrm{~mm}$ ), which had to be completely corrected. Also, radiographs had to be available (i.e. pre- and post-treatment panoramic radiographs and lateral cephalograms). Exclusion criteria were evidence of root resorptions on the pre-treatment panoramic radiographs, presence of severely dilacerated roots or endodontically treated teeth. Patients requiring other orthodontic systems, extraction therapy or any surgical treatments were also excluded.

In this practice, the radiographs were taken as a standard procedure for diagnostics before and at the end of active orthodontic treatment. No additional radiograph was conducted for this investigation.

To increase the aligner retention, additional attachments (i.e. tooth-coloured composite material) were applied as recommended by the manufacturer.

The anterior crowding was resolved by IPR (interproximal enamel reduction) and/or protrusion of the anterior teeth, based on the individual patient and determined by the orthodontist, depending on the initial overjet (protrusion) or shape of the tooth (IPR). The mean conducted IPR was $0.33 \mathrm{~mm}$ (Min. $0 \mathrm{~mm}$, Max. $0.5 \mathrm{~mm}$ ) per proximal contact.

Due to the primarily treatment issue, i.e. resolvement of anterior crowding, all upper and lower incisors and canines were assessed. Because of their use as anchorage during treatment, the first upper and lower molars were also assessed. Thus, a total of 1600 teeth were evaluated (400 upper, 400 lower incisors; 200 upper, 200 lower canines; 200 upper, 200 lower first molars). 


\section{Radiographic examinations}

All radiographs were taken with the same device. The measurement of the dental panoramic radiographs took place by using an electronic digital caliper rule (Studenroth GmbH Präzisionstechnik, Germany), calibrated in concordance with VDI/VDE/DGQ (Association of German Engineers/Association for Electrical, Electronic \& Information Technologies/German Society for Quality) guideline m2618 Sheet9.1 to an accuracy of $0.01 \mathrm{~mm}$. All root and crown measurements were taken twice in each radiograph and measured by one examiner blinded in a stochastic sequence.

To assess the pre- and post-treatment root length the following variables were measured under fourfold magnification on the basis of Fritz et al. [7] and Linge and Linge [10]: a symmetric cross was defined by two connecting lines. At first the long axis of each tooth was constructed by a line from the width of the incisal edge to the apex. The second line was defined by the connection of the mesial and distal cemento-enamel-junction. The crown length was declared as the distance between incisal edge and cemento-enamel-junction (on the long axis). The root length was defined as the distance between cemento-enamel-junction and apex (Figure 1).

\section{Analysis of the radiographs and virtual images}

The panoramic radiographs were as usual not standardized. Thus, errors like different projections or magnifications cannot be eliminated. But the crown-root length relation remains stable in different radiographs [7]. Therefore, we used the relation between the vertical root and crown length for further examination, which was described in another investigation [7] (Figure 1). Taking under consideration the pre-and post-treatment root and crown lengths, we calculated the individual root-crown-ratio (RCR) of each tooth and therefore the relative changes of RCR (rRCR) by a formula shown in Figure 1. An rRCR of $100 \%$ indicates no change of the pre-treatment root length relative to the post-treatment root length. A decrease of $\mathrm{rRCR}$ indicates a reduction of the root length during treatment.

To assess the real amount of orthodontic tooth movement in the front, we superimposed pre- and post-treatment lateral cephalograms. Sagittal and vertical alterations of the tooth position were measured in millimeters, as described in a previous investigation [7]. Vertical movement of the apex was categorized in extrusion or intrusion and sagittal movement in protrusion or retrusion. Tooth movement $\geq 1 \mathrm{~mm}$ was defined as relevant to the possible changes of RCR.

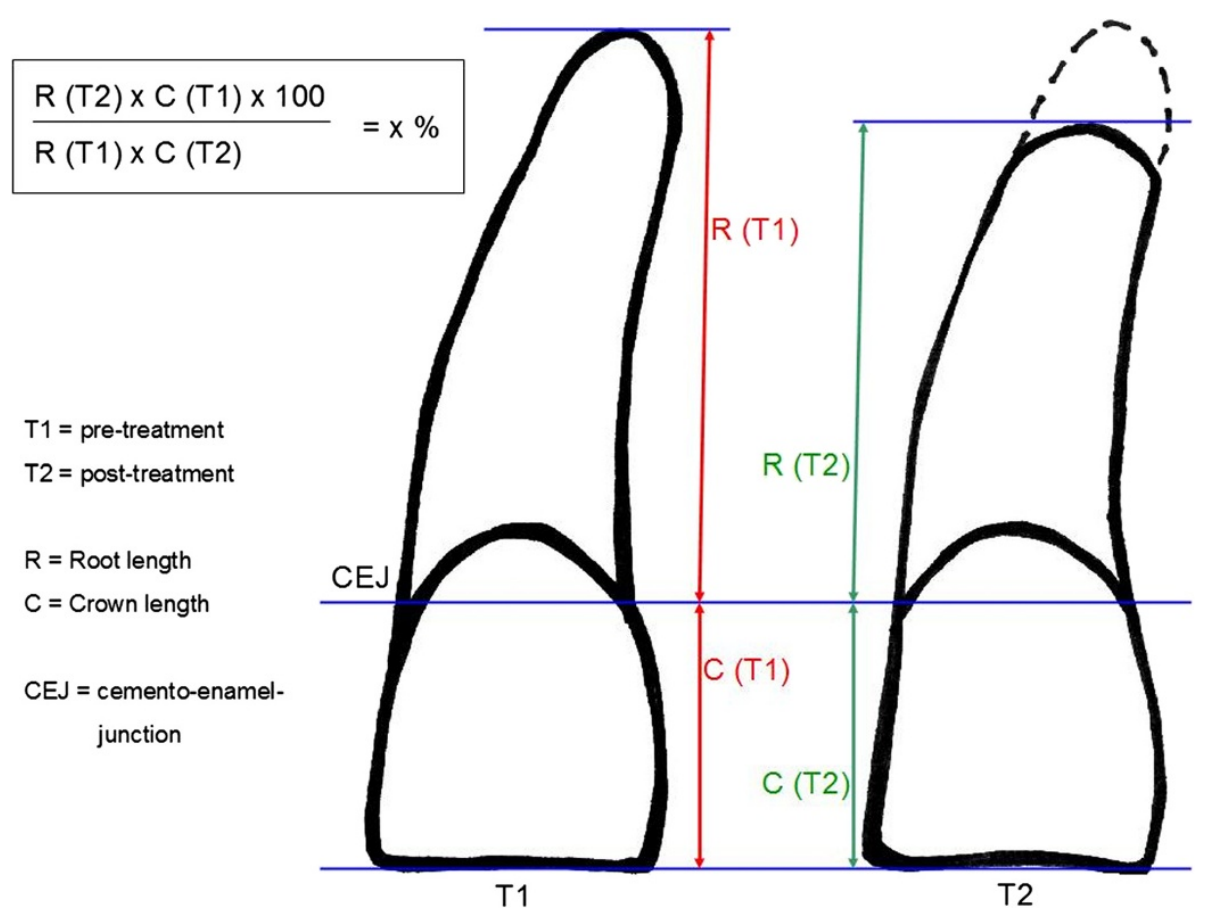

Figure 1 Measurement of the panoramic radiographs: root and crown lengths and the calculation-formula for the relative change of the root-crown-ratio $(\mathrm{RCR})$ during orthodontic treatment: post-treatment RCR relative to pre-treatment $\mathrm{RCR}$ ( $\mathrm{rRCR}$ ) in percentage; (CEJ = cemento-enamel junction) $[7,10]$. 
To assign the planned tooth movement of the front teeth, we examined sagittal and vertical movements in the individual 3-dimensional virtual images of each patient (ClinCheck ${ }^{\oplus}$, Align Technologies, Santa Clara, CA, USA). Previous studies showed a high accuracy between the virtual images and the treatment outcome $[19,20]$. Therefore, the images of before and at the end of treatment were compared and measured by a grid, set with the lowest adjustable scale ranges of $1 \mathrm{~mm}$.

\section{Statistical analysis}

Data analysis and data collection were performed using the SPSS software program (Statistical Package for Social Science) for Windows Version 18.0 (Inc., Chicago, II, USA) and the SAS software program, version 9.2 (Cary, NC).

The averages of the two measurements were used to compute RCR and the changes in RCR. We computed absolute and relative frequencies for categorical variables. Quantitative measurements are described by mean and standard deviation, minimum, maximum, median and quartiles. For testing changes in RCR we used $\log \left(\mathrm{RCR}_{\mathrm{t} 2 \mathrm{i}} /\right.$ $\left.\mathrm{RCR}_{\mathrm{t} 1 \mathrm{i}}\right)=\log \left(\mathrm{RCR}_{\mathrm{t} 2 \mathrm{i}}\right)-\log \left(\mathrm{RCR}_{\mathrm{t} 1 \mathrm{i}}\right)$ and performed a Hotelling $\mathrm{T}^{2}$ test. In order to assess a possible dependence of change in RCR on tooth movement we fitted a mixed linear model to $\log \left(\mathrm{RCR}_{\mathrm{t} 2 \mathrm{i}} / \mathrm{RCR}_{\mathrm{t} 1 \mathrm{i}}\right)$.

A Hotelling $\mathrm{T}^{2}$ test was performed to show significant changes in RCR during treatment. It gave a p-value of 0.0837 . Therefore, we fitted a linear mixed model to log (rRCR) including the following parameters as fixed effects: tooth position, intrusion, extrusion, retrusion, protrusion of upper jaw, intrusion, extrusion, retrusion, protrusion of lower jaw. We compared this model to a model only including an intercept term and a random patient effect.

\section{Method error}

Before the main measurements the examiner was calibrated by measuring ten different radiographs five times in random order and a minimum of two weeks in between the measurements.

For each tooth position and for both crown and root length we determined the components of variance -for inter-individual variation $\sigma_{\mathrm{A}}^{2}$ and the error variance $\sigma_{\mathrm{e}}^{2}$, this yields the intra-class-correlation ICC $=\sigma_{\mathrm{A}}^{2} /\left(\sigma_{\mathrm{A}}^{2}+\sigma_{\mathrm{e}}^{2}\right)$.

\section{Results}

\section{Calibration experiment}

The intra-class-correlation ranged from 0.845 (tooth 41) to 0.992 (tooth 33) for the crown measurements and from 0.929 (tooth 23) to 0.998 (tooth 43) for the root measurements, which indicated a high reproducibility and reliability.
Patient age, gender, treatment time and tooth position The patients were on average 37.7 (17-75) years of age, 37 males and 63 females with a mean treatment time of 19.8 months. On average, 32 aligners as well as 6 attachments were applied in the upper and lower jaw.

Analyzing these factors and the influence of the position of the tooth it turned out that none of these external factors was statistically significant correlated to the incidence of ARR (Table 1).

\section{Planned and real amount of tooth movement}

The planned tooth movement was analyzed by examination of the images $\left(\right.$ ClinCheck $\left.^{\odot}\right)$ before and the end of treatment. In most of the cases a retrusion of the incisors (52\% of all 100 patients) was planned in the upper jaw, mostly without vertical movements (43\%). Instead, in the lower jaw a protrusion (49\% of the cases) and intrusion (65\%) was planned.

Regarding the real amount the distribution is shown in Table 2. The mean tooth movement of the incisors ranged in the upper dentition from $0.52 \mathrm{~mm}$ (intrusion) up to $1.02 \mathrm{~mm}$ (retrusion), with minimum and maximum values of $0 \mathrm{~mm}$ up to $4 \mathrm{~mm}$. In the lower jaw the mean values ranged from $0.52 \mathrm{~mm}$ (intrusion) to 0.82 $\mathrm{mm}$ (protrusion), also with minimum and maximum values of $0 \mathrm{~mm}$ to $4 \mathrm{~mm}$. When looking at the F-tests for the individual parameters, it turned out that only the effect extrusion of the upper incisors can be considered as significant $(\mathrm{p}=0.0184)$ (Table 1$)$. On average, patients with extrusion of the upper incisors had only $96.1 \%$ of the rRCR of those patients without; i. e. extrusion of the upper incisors leads to more decrease in post-treatment RCRs than treatments without extrusion.

\section{Incidence and severity of ARR}

All patients had a minimum of two teeth affected with a reduction of the root length $(\mathrm{rRCR}<100 \%)$, on average 7.36 teeth per patient (Figure 2). $61 \%$ of all patients had a minimum of one tooth with a $20 \%$ root length reduction after treatment.

Table 1 Statistical analysis of the influence factors (n. s. = not significant)

\begin{tabular}{lc}
\hline Influence factors & Statistical significance \\
\hline Age & n. s. \\
Gender & n. s. \\
Individual tooth & n. s. \\
Change in RCR during treatment & n. s. \\
Sagittal tooth movement incisors & n. s. \\
Vertical tooth movement incisors & n. s. \\
Except: Extrusion of upper front teeth & $p=0.0184$ \\
\hline
\end{tabular}


Table 2 Distribution of vertical and sagittal orthodontic tooth movement of upper and lower incisors of every patient $(n=100)$, measured after superimposition of the pre- and post-treatment lateral cephalograms; mean, minimum and maximum values $[\mathrm{mm}], \mathrm{SD}=$ standard deviation

\begin{tabular}{|c|c|c|c|c|c|c|c|c|}
\hline & \multicolumn{2}{|c|}{ Protrusion } & \multicolumn{2}{|c|}{ Retrusion } & \multicolumn{2}{|c|}{ Extrusion } & \multicolumn{2}{|c|}{ Intrusion } \\
\hline & Upper & Lower & Upper & Lower & Upper & Lower & Upper & Lower \\
\hline Mean & 0.81 & 0.82 & 1.02 & 0.56 & 0.80 & 0.68 & 0.52 & 0.52 \\
\hline SD & 1.08 & 1.21 & 1.22 & 1.06 & 1.08 & 1.05 & 0.78 & 0.78 \\
\hline Min. & 0.00 & 0.00 & 0.00 & 0.00 & 0.00 & 0.00 & 0.00 & 0.00 \\
\hline Max. & 4.00 & 5.00 & 4.00 & 4.00 & 4.00 & 3.00 & 3.00 & 3.00 \\
\hline
\end{tabular}

$46 \%$ of the 1600 teeth $(n=736)$ presented a reduction of the post-treatment root length (rRCR $<100 \%$ ). Considering the severity, a reduction of $>0 \%$ up to $10 \%$ was found in $27.75 \%(\mathrm{n}=444)$, a distinct reduction of $>10 \%$ up to $20 \%$ in $11.94 \% \quad(n=191)$ of the sample. $6.31 \%(n=101)$ of the teeth were affected with a considerable reduction (>20\%) (Table 3 ). The values of the individual tooth (tooth 16, 13, 12, 11, 21, 22,-23, 26, 36, 33, 32, $31,41,42,43,46$; each $n=100$ ) are shown in Table 4 . To evaluate the extent of the maximum root length reduction during treatment, the distribution of the minimum rRCR values of all teeth are shown in Figure 3. On average, the minimum rRCR was $78.26 \%$. Only a few teeth were affected with a severe reduction of the root length.

\section{Discussion}

About the incidence of ARR in patients treated with aligners, there is only few reported evidence $[3,16]$. Therefore, this is the first study, investigating the incidence and severity of ARR in patients with a fulfilled aligner therapy.

ARR of upper incisors during treatment with aligners in a 25-year-old patient were described in a case report
[16]. Barbagallo et al. [17] investigated the effects of removable thermoplastic appliances (TA) vs. light or heavy forces with fixed appliances in premolars, which were afterwards extracted. They found that teeth, which experienced orthodontic tooth movement, had significantly more RR than the control teeth. TAs had similar RR as light (25 g) orthodontic forces [17]. But the treatment duration was only eight weeks and only sagittal movements were conducted. In the animal experimental study of Sombuntham et al. [18] clear plastic appliances were inserted but bonded to the residual dentition in rats. Their findings indicated initial superficial RR similar to $R R$ in another group of rats, induced by a closed coil spring. Due to the partly bonding, this force application might not be directly comparable with sequential removable appliances in humans [18]. Thermoplastic aligners are commonly removed several times a day e.g. food consumption.

Practically all patients had ARR. Every patient had a minimum of two teeth affected with a reduction of the root length after treatment. Two recent studies also investigated ARR in patients with a class I occlusion and anterior crowding, but treated with multibracket

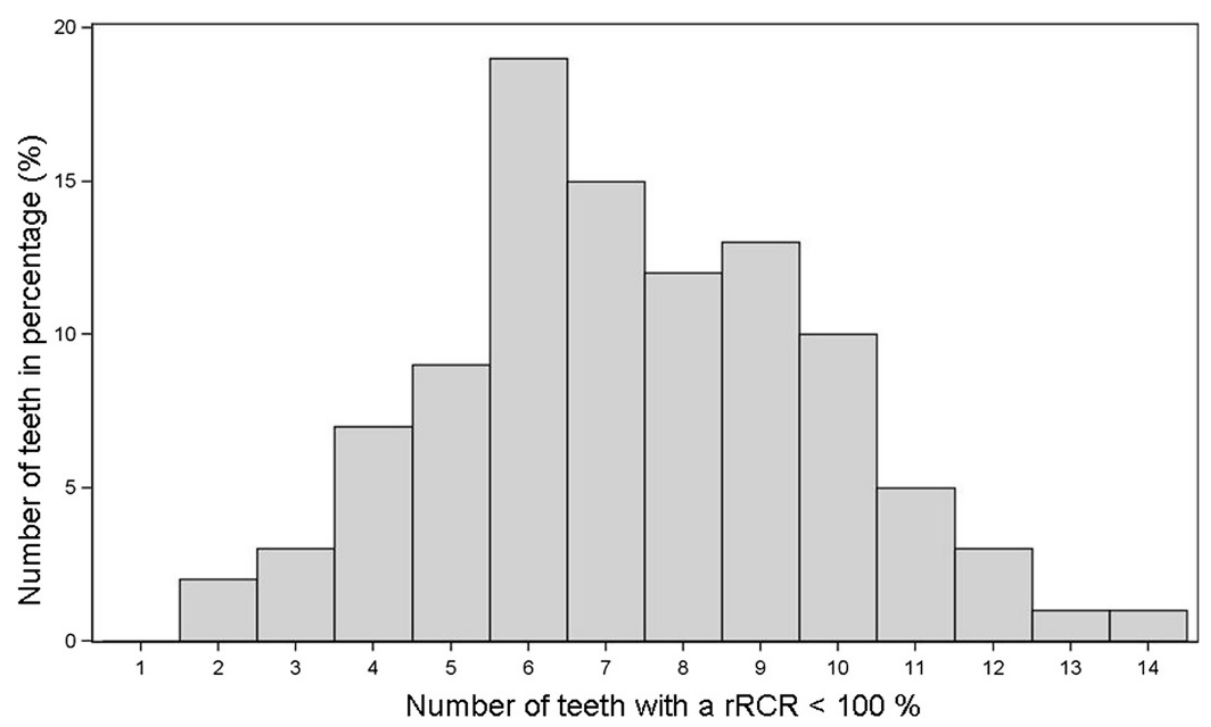

Figure 2 Distribution of the amount of the affected teeth per patient $(n=100)$. 
Table 3 Distribution of the root length reduction during orthodontic treatment: post-treatment $\mathrm{RCR}$ relative to pre-treatment RCR (rRCR), absolute (n) and relative (\%) frequencies $(n=1600)$ : $r R C R<80$ means a reduction over $20 \%$ of pre-treatment root length

\begin{tabular}{ccc}
\hline rRCR & $\begin{array}{c}\text { Frequency } \\
(\mathbf{n})\end{array}$ & $\begin{array}{c}\text { Percent } \\
\text { (\%) }\end{array}$ \\
\hline $\mathrm{rRCR}<80$ & 101 & 6.31 \\
$80 \leq \mathrm{rRCR}<90$ & 191 & 11.94 \\
$90 \leq \mathrm{rRCR}<100$ & 444 & 27.75 \\
$\mathrm{rRCR} \geq 100$ & 864 & 54.00 \\
\hline
\end{tabular}

appliances. They assessed RR with bone beam computed tomography $(\mathrm{CBCT})$ and reported about similar results as the present study $[11,12]$. $46 \%$ of all teeth in our study presented a root reduction. This is in concordance with Castro et al. [11], who reported also about an incidence of $46 \%$. Lund et al. [12] instead described that $91 \%$ of all teeth showed some degree of ARR $(<1 \mathrm{~mm})$. This may due to the fact, that the patients in their study were treated with an extraction of one premolar in each jaw quadrant for resolving the anterior crowding. Therefore, the treatment was more extensive than in our study or Castro et al. [11], where no extraction was performed. For the first time we showed that the incidence of ARR in patients treated with aligners was similar to previous studies analyzing the incidence of ARR in patients with multibracket-appliances when resolving anterior crowding.

Regarding influence factors, we found no statistically significant association between the relative changes of RCR and age and gender, which is in concordance with Castro et al. [11]. An association between the root length reduction and the individual tooth could also not be found. Other studies instead reported about most significant ARR and highest frequencies in incisors and first molars. Commonly, maxillary incisors develop ARR more often and more severely than other teeth [6-12]. We found a statistically higher RR in maxillary incisors after extrusion but the value was clinically negligible due to the small mean value.

We also observed no statistical correlation to sagittal and vertical orthodontic tooth movement, except for extrusion of upper incisors. A finite element study demonstrated that the highest amount of stress was produces by vertical forces e.g. extrusion in the apical region [21]. A previous study investigating intrusive and extrusive force applications found that intrusive forces significantly increased RR rates compared with the controls and extrusive forces were not significantly different [22]. In treatments with removable aligners vertical movements are more difficult to perform $[19,20,23]$. Therefore, they have to be conducted with attachments or other auxillaries like elastics. It could be assumed that those force applications are not very precisely to apply, what might be a reason for causing RR, or the magnitude of the applied extrusive forces was too high. But it should be investigated in a greater sample for more details.

Regarding the clinical relevance of RR in the apical region, the working group of Kalkwarf et al. [24] described that a loss of alveolar bone in the marginal area, caused by periodontal diseases, has more influence on the periodontal support than ARR. Even with an ARR of $3 \mathrm{~mm}$ the periodontal attachment still remained $87.10 \%$ [24]. They reported that $3 \mathrm{~mm}$ of ARR were nearly equivalent to $1 \mathrm{~mm}$ of marginal bone loss [24]. Therefore, with regards to the small amount (average reduction of the root length of $4 \%$ ) and the possibility of being an artefact, the factor extrusion was considered as not clinical relevant.

The first molars, which were less intentionally moved, had also a reduction of the root length. This is in

Table 4 Distribution of the root length reduction during orthodontic treatment of the individual teeth (upper and lower incisors, canines, first molars) (each $n=100$, overall $n=1600$ ): post-treatment $R C R$ relative to pre-treatment $R C R$ (rRCR), absolute $(n)$ and relative $(\%)$ frequencies

\begin{tabular}{|c|c|c|c|c|c|c|c|c|c|c|c|c|c|c|c|c|}
\hline \multirow[b]{2}{*}{$\mathrm{rRCR}$} & \multicolumn{16}{|c|}{ Tooth } \\
\hline & 16 & 13 & 12 & 11 & 21 & 22 & 23 & 26 & 46 & 43 & 42 & 41 & 31 & 32 & 33 & 36 \\
\hline \multirow[t]{2}{*}{$\mathrm{rRCR}<80$} & 7 & 12 & 6 & 10 & 7 & 2 & 7 & 8 & 1 & 2 & 1 & 10 & 14 & 5 & 6 & 3 \\
\hline & 7.0 & 12.0 & 6.0 & 10.0 & 7.0 & 2.0 & 7.0 & 8.0 & 1.0 & 2.0 & 1.0 & 10.0 & 14.0 & 5.0 & 6.0 & 3.0 \\
\hline \multirow[t]{2}{*}{$80 \leq r R C R<90$} & 11 & 11 & 15 & 11 & 12 & 11 & 16 & 8 & 9 & 9 & 18 & 15 & 12 & 11 & 7 & 14 \\
\hline & 11.0 & 11.0 & 15.0 & 11.0 & 12.0 & 11.0 & 16.0 & 8.0 & 9.0 & 9.0 & 18.0 & 15.0 & 12.0 & 11.0 & 7.0 & 14.0 \\
\hline \multirow[t]{2}{*}{$90 \leq \mathrm{rRCR}<100$} & 22 & 24 & 28 & 22 & 22 & 29 & 27 & 36 & 38 & 31 & 25 & 27 & 25 & 32 & 24 & 33 \\
\hline & 22.0 & 24.0 & 28.0 & 22.0 & 22.0 & 29.0 & 27.0 & 36.0 & 38.0 & 31.0 & 25.0 & 27.0 & 25.0 & 32.0 & 24.0 & 33.0 \\
\hline \multirow[t]{2}{*}{$r R C R \geq 100$} & 60 & 53 & 51 & 57 & 59 & 58 & 50 & 48 & 52 & 58 & 56 & 48 & 49 & 52 & 63 & 50 \\
\hline & 60.0 & 53.0 & 51.0 & 57.0 & 59.0 & 58.0 & 50.0 & 48.0 & 52.0 & 58.0 & 56.0 & 48.0 & 49.0 & 52.0 & 63.0 & 50.0 \\
\hline Total (n) & 100 & 100 & 100 & 100 & 100 & 100 & 100 & 100 & 100 & 100 & 100 & 100 & 100 & 100 & 100 & 100 \\
\hline
\end{tabular}




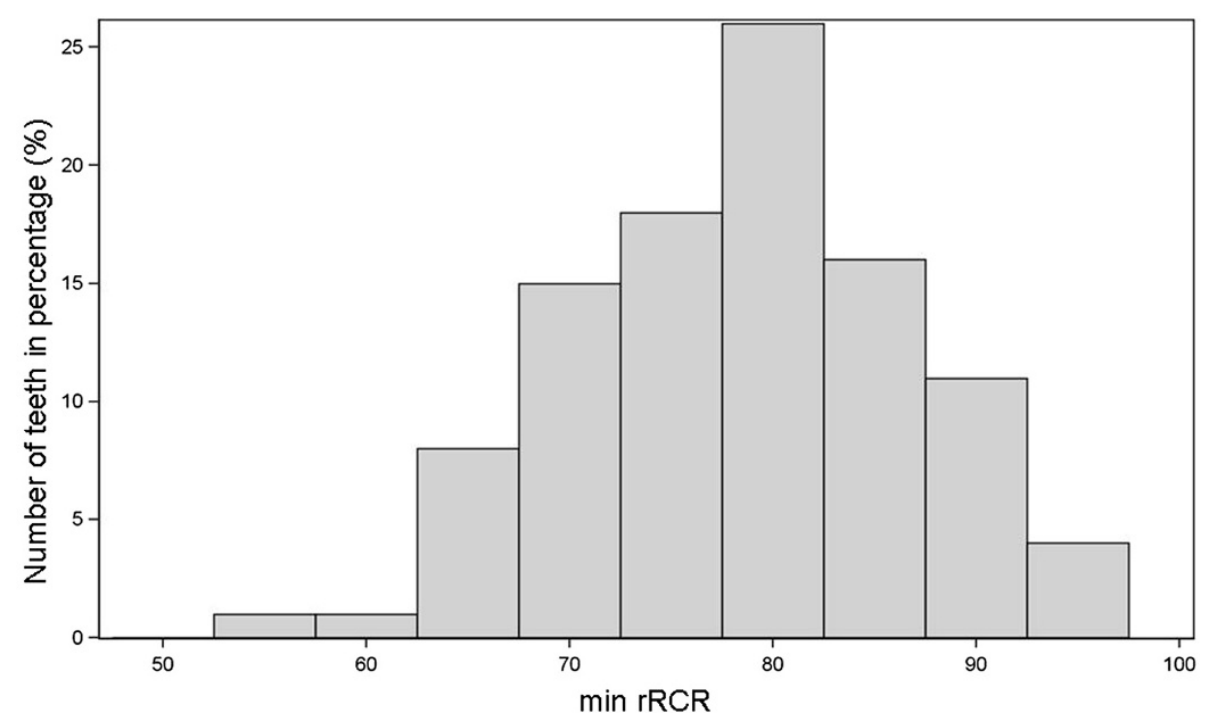

Figure 3 Distribution of the maximum root length reduction, i.e. minimum rRCR (= min rRCR), of each individual tooth $(n=1600)$.

concordance with Castro et al. [11], who also found ARR in molars, when resolving anterior crowding patients with a class I occlusion (using multibracket appliances). Therefore, we concluded that using teeth as an anchorage e.g. with attachments or even being included in the arch where an aligner is inserted might deliver enough force to cause RR.

To measure and assess RR, 2-dimensional radiographs are still presently a common method [13,25]. A striking limitation of this method is that only ARR can be measured. 3-dimensional techniques instead obtain more accurate measurements and give the opportunity to assess the entire root length 3-dimensionally. The degree of ARR seems to be underestimated in panoramic radiographs compared to CBCT [9], when using a scoring system like Levander and Malmgren [9]. Instead of measuring metrically the tooth length in 2-dimensional panoramic radiographs many studies classified changes of the root length in resorption stages [13,26]. We evaluated the relative change of RCR in percentage. RCR was considered stable, even if the two measured radiographs of each patient have different scales (projection-related errors) [7].

In recent studies, the higher reliability and accuracy of CBCT revealing even small amounts of ARR are shown $[11,12]$. But still, CBCT have not replaced panoramic radiographs, which remains the primary imaging modality. CBCTs have a higher radiation exposure with a greater medical risk for the patient [27]. The effective doses of CBCTs can be 1.5 to 33 times higher than panoramic radiographs [28].

A limit of this investigation is the fact that it is a retrospective study. But due to the stringent inclusion criteria, the fact that all patient were treated by the same practitioner, and all radiographs taken by the same device, offers this study reliable results with a high number of patients.

\section{Conclusion}

The present study is the first analyzing apical roots resorptions in patients with a fully implemented orthodontic treatment with aligners. Every patient had a minimum of two teeth with root length reduction when resolving anterior crowding. On average, 7.36 teeth per patient were affected. Overall, 54\% of the measured 1600 teeth showed signs of apical root resorption, $6.31 \%$ a reduction of over $20 \%$ of the pre-treatment root length. No clinical relevant influence factor could be detected. Therefore, due to the high individual variability in the degree of the occurred root resorptions, there is no prediction by external influence factors.

\section{Abbreviations}

ARR: Apical root resorption; RR: Root resorption; RCR: Root-crown-ratio; rRCR: Relative root-crown-ratio.

\section{Competing interests}

The authors declare that they have no conflict of interest.

\section{Authors' contributions}

EK carried out the conception of the study. She supervised the measurements, assembled the data, conducted the interpretation of the data, and drafted the manuscript. TD provided the patient pool from which the sample was selected. IS analyzed the data and helped with the manuscript. CJ was involved in conception and design of the study, also in analysis and interpretation of data, and drafting the manuscript. SH collected the data and conducted the measurements. HW conceived of the study, and participated in its design and coordination and helped to draft the manuscript. All authors read and approved the final manuscript.

\section{Author details}

${ }^{1}$ Department of Orthodontics, Medical Centre of the Johannes-Gutenberg-University Mainz, Augustusplatz 2, 55131 Mainz, Germany. ${ }^{2}$ Private practice, Wilhelmstraße 40, 
65183 Wiesbaden, Germany. ${ }^{3}$ Institute of Medical Biostatistics, Epidemiology and Informatics, University Medical Centre of the Johannes-Gutenberg-University Mainz, Obere Zahlbacher Str. 69, 55131 Mainz, Germany.

Received: 10 July 2013 Accepted: 9 August 2013

Published: 14 August 2013

\section{References}

1. Weltman B, Vig KW, Fields HW, Shanker S, Kaizar EE: Root resorption associated with orthodontic tooth movement: a systematic review. Am J Orthod Dentofacial Orthop 2010, 137:462-476.

2. Al-Qawasmi RA, Hartsfield JK Jr, Everett ET, Flury L, Liu L, Foroud TM, Macri JV, Roberts WE: Genetic predisposition to external apical root resorption Am J Orthod Dentofacial Orthop 2003, 123:242-252.

3. Killiany DM: Root resorption caused by orthodontic treatment: an evidence-based review of literature. Semin Orthod 1999, 5:128-133.

4. Kurol J, Owman-Moll P: Hyalinization and root resorption during early orthodontic tooth movement in adolescents. Angle Orthod 1998, 68:161-165.

5. Wehrbein $H$, Harhoff $R$, Diedrich P: Rates of root resorption in orthodontically moved, periodontally affected and healthy teeth. Dtsch Zahnarztl Z 1990, 45:176-178 [in German].

6. Brezniak N, Wasserstein A: Root resorption after orthodontic treatment: Part 1. Literature review. Am J Orthod Dentofacial Orthop 1993, 103:62-66.

7. Fritz $U$, Diedrich $P$, Wiechmann D: Apical root resorption after lingual orthodontic therapy. J Orofac Orthop 2003, 64:434-442.

8. Gülden N, Eggermann T, Zerres K, Beer M, Meinelt A, Diedrich P: Interleukin-1 polymorphisms in relation to external apical root resorption (EARR). J Orofac Orthop 2009, 70:20-38.

9. Levander $\mathrm{E}$, Malmgren O: Evaluation of the risk of root resorption during orthodontic treatment. A study of upper incisors. Eur J Orthod 1988, 10:30-38.

10. Linge $B O$, Linge L: Apical root resorption in upper anterior teeth. Eur J Orthod 1983, 5:173-183.

11. Castro IO, Alencar AH, Valladares-Neto J, Estrela C: Apical root resorption due to orthodontic treatment detected by cone beam computed tomography. Angle Orthod 2012, 83:196-203.

12. Lund $\mathrm{H}$, Gröndahl K, Hansen K, Gröndahl HG: Apical root resorption during orthodontic treatment. A prospective study using cone beam CT. Angle Orthod 2012, 82:480-487.

13. Sehr K, Bock NC, Serbesis C, Hönemann M, Ruf S: Severe external apical root resorption-local cause or genetic predisposition? J Orofac Orthop 2011, 72:321-331.

14. Bastos Lages EM, Drummond AF, Pretti H, Costa FO, Lages EJ, Gontijo Al, Miranda Cota LO, Brito RB Jr: Association of functional gene polymorphism IL-1b in patients with external apical root resorption. Am J Orthod Dentofacial Orthop 2009, 136:542-546.

15. Harris EF, Kineret SE, Tolley EA: A heritable component for external apical root resorption in patients treated orthodontically. Am J Orthod Dentofacial Orthop 1997, 111:301-309.

16. Brezniak $\mathrm{N}$, Wasserstein $\mathrm{A}$ : Root resorption following treatment with aligners. Angle Orthodontist 2008, 78:1119-1124.

17. Barbagallo LJ, Jones AS, Petocz P, Darendeliler MA: Physical properties of root cementum: part 10. Comparison of the effects of invisible removable thermoplastic appliances with light and heavy orthodontic forces on premolar cementum. A microcomputed-tomography study. Am J Orthod Dentofacial Orthop 2008, 133:218-227.

18. Sombuntham NP, Songwattana $S$, Atthakorn $P$, Jungudomjaroen $S$, Panyarachun B: Early tooth movement with a clear plastic appliance in rats. Am J Orthod Dentofacial Orthop 2009, 136:75-82.

19. Krieger E, Seiferth J, Marinello I, Jung BA, Wriedt S, Jacobs C, Wehrbein H: Invisalign ${ }^{\oplus}$ treatment in the anterior region-were the predicted tooth movements achieved? J Orofac Orthop 2012, 73:365-376.

20. Krieger E, Seiferth J, Saric I, Jung BA, Wehrbein H: Accuracy of Invisalign ${ }^{\circledR}$ treatments in the anterior tooth region. First results. J Orofac Orthop 2011, 72:141-149.

21. Rudolph DJ, Willes PMG, Sameshima GT: A finite element model of apical force distribution from orthodontic tooth movement. Angle Orthod 2001 71:127-131.
22. Han G, Huang S, Von den Hoff JW, Zeng X, Kuijpers-Jagtman AM: Root resorption after orthodontic intrusion and extrusion: an intraindividual study. Angle Orthod 2005, 75:912-918.

23. Kravitz ND, Kusnoto B, BeGole E, Obrez A, Agran B: How well does Invisalign ${ }^{\oplus}$ work? A prospective clinical study evaluating the efficacy of tooth movement with Invisalign ${ }^{\circledR}$. Am J Orthod Dentofacial Orthop 2009, 135:27-35.

24. Kalkwarf KL, Krejci RF, Pao YC: Effect of apical root resorption on periodontal support. J Prosthet Dent 1986, 56:317-319.

25. Apajalahti S, Peltola JS: Apical root resorption after orthodontic treatment - a retrospective study. Eur J Orthod 2007, 29:408-412.

26. Janson GR, De Luca Canto G, Martins DR, Henriques JF, De Freitas MR: A radiographic comparison of apical root resorption after orthodontic treatment with 3 different fixed appliance techniques. Am J Orthod Dentofacial Orthop 2000, 118:262-273.

27. Dudic A, Giannopoulou C, Leuzinger M, Kiliaridis S: Detection of apical root resorption after orthodontic treatment by using panoramic radiography and cone-beam computed tomography of super-high resolution. Am J Orthod Dentofacial Orthop 2009, 135:434-437.

28. Silva MA, Wolf U, Heinicke F, Bumann A, Visser H, Hirsch E: Cone-beam computed tomography for routine orthodontic treatment planning: a radiation dose evaluation. Am J Orthod Dentofacial Orthop 2008, 133:640. e1-640.e5.

doi:10.1186/1746-160X-9-21

Cite this article as: Krieger et al:: Apical root resorption during orthodontic treatment with aligners? A retrospective radiometric study. Head \& Face Medicine 2013 9:21

\section{Submit your next manuscript to BioMed Central and take full advantage of:}

- Convenient online submission

- Thorough peer review

- No space constraints or color figure charges

- Immediate publication on acceptance

- Inclusion in PubMed, CAS, Scopus and Google Scholar

- Research which is freely available for redistribution 